\title{
Calibration of Johnson's damage model by a Bayesian approach
}

\author{
Guillaume Perrin ${ }^{1, *}$ and Laurianne Pillon ${ }^{1, * *}$ \\ ${ }^{1}$ CEA, DAM, DIF, F-91297 Arpajon Cedex, France
}

\begin{abstract}
A calibration of the Johnson's damage model is proposed by using a Bayesian approach. A set of 3 parameters has to be adjusted. For this purpose, free surface velocities of 7 plate impact experiments leading to spallation in targets of tantalum are used. The resulting distributions of probability indicate several potential values for each parameters. Each of them used in an hydrocode leads to good agreement with reference free surface velocities.
\end{abstract}

\section{Introduction}

Spallation is one of the major mechanisms encountered in dynamic experiments [1]. It occurs after an intense and local tensile loading in the material. Spalling rupture consists in a physical separation of matter among a plane parallel to the propagation of the shock front. In ductile materials, damage and rupture events are governed by plastic deformation around voids that nucleate, grow and join each other in order to form a macroscopic crack called spall.

Hollow sphere models allow an accurate description of void growth in spall experiments [2,3]. They introduce a minimal number of parameters, adjusted by comparison to experiments dedicated to damage observation. The purpose of this article is to propose a method for the adjustment of these parameters. We use a Bayesian approach [4-6] and a comparison to dedicated experiments.

In the first part of this paper, we remind principles of calibration methods, in the second part, we present the damage model used for this calibration and finally in the third part, we present experiments used as a reference to adjust parameters of the model and results obtained by the calibration method.

\section{Calibration method and potential sources of uncertainties}

We consider a physical phenomenon appearing in several experiments, characterized by an input vector $\mathbf{x}$ containing specific quantities. The physical phenomenon can be described by a model containing unknown parameters, defining the vector $\beta$. This physical modeling is used in simulations and must provide an accurate description of the experiments. Specific quantities, obtained from experimental results, are summarized in a vector $\mathbf{Y}$. For a given configuration $\mathbf{x}$, the vector $\mathbf{Y}$ can be evaluated by different ways:

\footnotetext{
*e-mail: guillaume.perrin2@cea.f

**e-mail: laurianne.pillon@cea.fr
}

- the real value $\mathbf{Y}^{\text {real }}(\mathbf{x})$, which is unknown,

- the measurement $\mathbf{Y}^{\text {mes }}(\mathbf{x})$

- and the value deduced by the model $\mathbf{Y}^{\text {code }}(\mathbf{x}, \boldsymbol{\beta})$.

The calibration aims at identifying the most relevant parameters vector $\boldsymbol{\beta}$ in order to describe at best the experimental data base.

When a measurement is performed, there is some experimental error $\mathbf{Y}^{\text {mes }}(\mathbf{x})=\mathbf{Y}^{\text {real }}(\mathbf{x})+\boldsymbol{\epsilon}^{\text {mes }}(\mathbf{x})$. When a physical phenomenon is described by simulations, a lack of precision comes from the approximation of the physical modeling and from the weakness of the numerical resolution. They can be summarized in a model error $\mathbf{Y}^{\text {real }}(\mathbf{x})=\mathbf{Y}^{\text {code }}(\mathbf{x}, \boldsymbol{\beta})+\boldsymbol{\epsilon}^{\mathrm{mod}}(\mathbf{x})$. Finally, the difference between measured and described quantities is defined by:

$$
\mathbf{Y}^{\text {mes }}(\mathbf{x})-\mathbf{Y}^{\text {code }}(\mathbf{x}, \boldsymbol{\beta})=\boldsymbol{\epsilon}^{\text {mod }}(\mathbf{x})+\boldsymbol{\epsilon}^{\text {mes }}(\mathbf{x})
$$

This quantity is infered by using the Bayesian approach.

\section{Damage model for the calibration}

In this study, we use the above presented calibration method to adjust results obtained by a damage model describing spallation in ductile materials : the Johnson's damage model [3].

Spalling rupture is defined as a fracture in a material induced by an intense and local tensile state. At the macroscopic scale, this phenomenon results from the creation of voids that grow and join each other in order to form a macroscopic crack. The Johnson's model is an hollow sphere model. It describes the growth of voids in a ductile viscoplastic material. The rate of growth of voids, characterized by a porosity variable $\alpha$, is defined with respect to the pressure $P$ :

$$
\begin{array}{cc}
\text { if } & \Delta P \leq 0, \quad \dot{\alpha}=0 \\
\text { if } & \Delta P>0, \quad \dot{\alpha}= \\
& -\operatorname{sign}(P) \frac{\left(\alpha_{0}-1\right)^{2 / 3}}{\eta} \alpha(\alpha-1)^{1 / 3} \Delta P
\end{array}
$$


with $\Delta P=|P|-P_{\text {eq }}$ and $\eta$ the material viscosity. An equilibrium pressure is defined by $P_{\text {eq }}=A_{\mathrm{s}} / \alpha \log (\alpha /(\alpha-1))$ with $A_{\mathrm{s}}$ a parameter, proportional to the yield stress in the material.

The growth phase begins with an initial porosity $\alpha_{0}$ and ends when the porosity $\alpha$ reaches a maximum porosity $\alpha_{\mathrm{L}}$. When porosity exceeds this parameter, the material is considered as locally broken. It is then described by a rupture modeling.

Three parameters drive the description of damage provided by Johnson's model. They define the parameters vector to adjust: $\beta=\left(A_{\mathrm{s}}, \eta, \alpha_{\mathrm{L}}\right)$. This is the topic of the next section.

\section{Results of the calibration and comparison with experiments}

In order to perform the calibration of $\boldsymbol{\beta}$, plate impact experiments are used [7]. In these experiments, a projectile plate, named "impactor", is projected with a velocity $V_{\mathrm{P}}$ by a powder gun. This plate hits a target initially at rest. After the impact of the projectile on the target, shock waves are generated in the impactor and the target, which reflect on free surfaces in release waves. A sudden tensile state is generated when release waves cross each other. Damage rapidly grows until local rupture appears. Damage occurring in this target is characterized in such experiments.

For the calibration, $N=7$ plate impact experiments are used. All the experiments use aluminium impactors and tantalum targets. Each experiment is defined by three data: the thickness of the target in tantalum $e_{\mathrm{T}}$, the thickness of the projectile in aluminium $e_{\mathrm{P}}$ and the velocity of the projectile impacting the target $V_{\mathrm{P}}$. These three variables define the input vector $\mathbf{x}=\left(V_{\mathrm{P}}, e_{\mathrm{T}}, e_{\mathrm{P}}\right)$. Target diameters are not given in this study since the phenomenon is described in one dimension. In these experiments, the

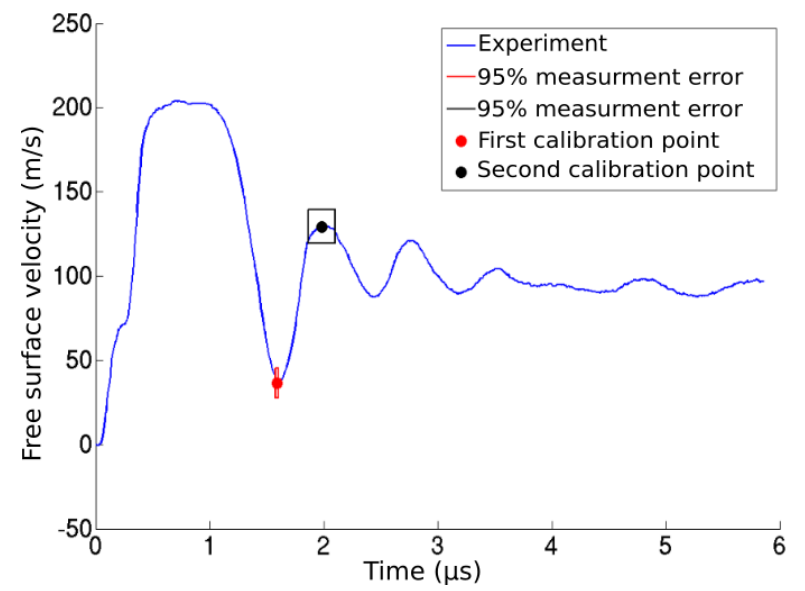

Fig. 1. Typical evolution of the free surface velocity.

velocity component normal to the free surface of the target is recorded at the center of the target. The free surface velocity obtained in the first experiment of the series used for the calibration is plotted in figure 1. Its evolution
$V\left(t_{i}\right), 1 \leq i \leq N$ is typical of the one obtained in a spall experiment. The overall evolution of the free surface velocity can be characterized by a small number of specific points. For this study, the following points have been chosen:

1. the first local minimum $\left(t^{\mathrm{min}}, V\left(t^{\mathrm{min}}\right)\right)$ depends on spall opening (spatial position and time at which the crack is created),

2. the second local maximum $\left(t^{\max }, V\left(t^{\max }\right)\right)$ depends on the description of the opened crack and of shock waves in spall.

They are indicated on the example of the figure 1. Coordinates of these two points define the vector $\mathbf{Y}$, called quantities of interest vector. These two points depend on damage evolution leading to spall opening and then on parameters of Johnson's damage model. A calibration analy-
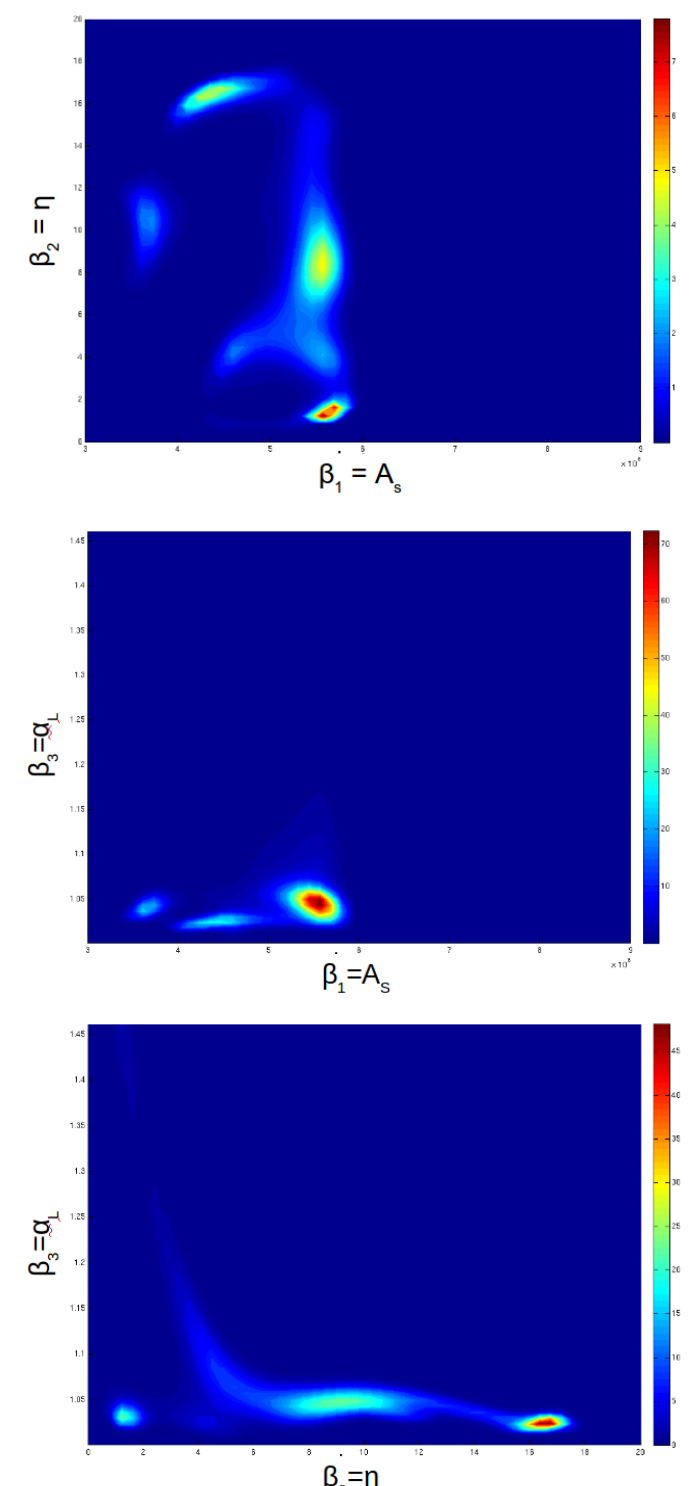

Fig. 2. 2D-representation of the distribution of parameters $\boldsymbol{\beta}$ provided by the calibration procedure.

sis has been performed by using an hydrodynamical code. 
The parameters distribution with an estimation of the uncertainty can be evaluated too. A 2D-representation of this distribution is plotted in figure 2 .

The obtained distribution is not gaussian. Several areas with high probability can be found, which indicates that several set of parameters are realistic. This observation is usual. In the table 1 have been reported the values at the center of the areas of maximum probability.

Table 1. Most probable set of parameters.

\begin{tabular}{|c|c|c|c|}
\hline$\beta_{1}=A_{s}(\mathrm{MPa})$ & $\beta_{2}=\eta(\mathrm{Pa} . \mathrm{s})$ & $\beta_{3}=\alpha_{L}$ & Probability \\
\hline 434 & 16.3 & 1.02 & $30 \%$ \\
\hline 557 & 8.57 & 1.04 & $40 \%$ \\
\hline 569 & 1.63 & 1.03 & $20 \%$ \\
\hline 374 & 10.6 & 1.04 & $10 \%$ \\
\hline
\end{tabular}

Results obtained by 10 likely values of the vector $\beta$ have been plotted in the figure 3. A good agreement with experimental results is obtained for each of this set of parameters. We can mention that the parameter $\beta_{2}=\eta$ has a weak influence on this results, which indicates a weak influence of this parameter on Johnson's damage description. It is probably due to experiments which are not restrictive enough. Another hypothesis is that it comes from the modeling: a similar study on another material should indicate if this trend is due to the damage model or to the description of tantalum. This study induces several

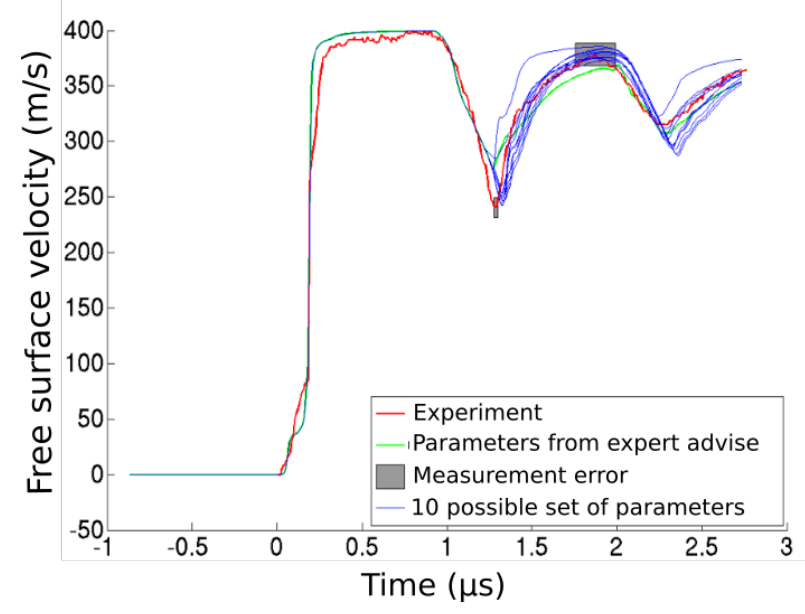

Fig. 3. Description of the experiment number 4 by Johnson's damage model with 10 values of parameters vector $\beta$.

choices. First, the choice of the quantities of interest char- acterizing the experimental data base can be discussed. Another typical point could have been added, in particular, the second local minimum on the free surface velocity. Secondly, other quantities could have been included in the input vector $\mathbf{x}$ or other experiments could be considered too. At least, introducing prior information coming from other experiments would restrict the calibration. They can be deduced from other dedicated experiments.

\section{Conclusion}

A calibration method has been used in order to adjust Johnson's damage model, which allows a description of spalling rupture in targets of tantalum. For this purpose, a series of 7 basic spall experiments has been used. Several sets of parameters have been identified by this method. A good agreement with experimental data is obtained with these different parameters.

Bayesian approaches are currently more and more used in several domains of physics. They allow to overcome usual methods used for the adjustment of models in which a unique set of parameters is searched for. Bayesian approaches lead to a distribution of probability for each parameter. The most probable set of parameters give a good agreement with an ensemble of experimental data. They allow to quantify uncertainties associated. This type of method is very interesting for the calibration of physics models in the future.

\section{References}

1. B. Hopkinson, Trans. R. Soc. London 213A, p. 437 (1914)

2. M. N. Carroll and A. C. Holt, J. Appl. Phys. 43, p. 1626 (1972)

3. J. N. Johnson, J. Appl. Phys. 52, p. 2812-2825 (1981)

4. G. E. Box and G. C. Tiao, Bayesian inference in statistical analysis, (Wiley, 1973)

5. M. Kennedy and A. O'Hagan, Journal of the Royal Statistical Society, 63, p. 425-464 (2001)

6. T. Hastie, R. Tibshirani, J. Friedman, The elements of statistical learning: data mining, inference and prediction, (Springer, New York, 2001)

7. M. Nicollet, P. Manczur, J. C. Redasse and C. Rion, Private communication (2001) 
